\title{
A WEAK ERGODIC THEOREM FOR INFINITE PRODUCTS OF LIPSCHITZIAN MAPPINGS
}

\author{
SIMEON REICH AND ALEXANDER J. ZASLAVSKI
}

Received 16 May 2002

Let $K$ be a bounded, closed, and convex subset of a Banach space. For a Lipschitzian self-mapping $A$ of $K$, we denote by $\operatorname{Lip}(A)$ its Lipschitz constant. In this paper, we establish a convergence property of infinite products of Lipschitzian self-mappings of $K$. We consider the set of all sequences $\left\{A_{t}\right\}_{t=1}^{\infty}$ of such selfmappings with the property $\lim \sup _{t \rightarrow \infty} \operatorname{Lip}\left(A_{t}\right) \leq 1$. Endowing it with an appropriate topology, we establish a weak ergodic theorem for the infinite products corresponding to generic sequences in this space.

\section{Introduction}

The asymptotic behavior of infinite products of operators finds applications in many areas of mathematics (see, e.g., $[1,2,3,4,5,8,9,10,12,14,15,16,17,18]$ and the references therein). Given a bounded, closed, and convex subset $K$ of a Banach space and a sequence $\mathbf{A}=\left\{A_{t}\right\}_{t=1}^{\infty}$ of self-mappings of $K$, we are interested in the convergence properties of the sequence of products $\left\{A_{n} \cdots A_{1} x\right\}_{n=1}^{\infty}$, where $x \in K$. In the special case of a constant sequence $\mathbf{A}$, we are led to study the asymptotic behavior of a single operator. In their seminal paper [7], De Blasi and Myjak show that the powers of a generic nonexpansive self-mapping of $K$ do converge. Such an approach, when a certain property is investigated for a whole space of operators and not just for a single operator, has already been successfully applied in many areas of analysis. For instance, in a recent paper [15], we have extended the De Blasi-Myjak result in several directions to certain sequence spaces of nonexpansive operators. One of these directions has involved weak ergodicity in the sense of population biology (see $[6,11,13,15])$. More precisely, we have shown that for most (in the sense of Baire's categories) sequences, the distances between the corresponding (random) infinite products with different initial points tend to zero, uniformly on $K$. The main result of 
the present paper (Theorem 1.1 below) is an extension of [15, Theorem 2.2] to Lipschitzian mappings which are not necessarily nonexpansive.

Assume that $(X,\|\cdot\|)$ is a Banach space and that $K \subset X$ is a bounded, closed, and convex subset of $X$.

For any $A: K \rightarrow X$, define

$$
\operatorname{Lip}(A)=\sup \{\|A x-A y\| /\|x-y\|: x, y \in K \text { and } x \neq y\} .
$$

Denote by $\mathscr{A}$ the set of all sequences $\mathbf{A}=\left\{A_{t}\right\}_{t=1}^{\infty}$, where each $A_{t}: K \rightarrow K$ satisfies $\operatorname{Lip}\left(A_{t}\right)<\infty, t=1,2, \ldots$, and

$$
\limsup _{t \rightarrow \infty} \operatorname{Lip}\left(A_{t}\right) \leq 1
$$

Set

$$
d(K)=\sup \{\|x-y\|: x, y \in K\}
$$

For $\mathbf{A}=\left\{A_{t}\right\}_{t=1}^{\infty}$ and $\mathbf{B}=\left\{B_{t}\right\}_{t=1}^{\infty}$ in $\mathscr{A}$, define

$$
\begin{aligned}
d_{s}(\mathbf{A}, \mathbf{B})= & \sup \left\{\left\|A_{t} x-B_{t} x\right\|: t=1,2, \ldots \text { and } x \in K\right\} \\
& +\sup \left\{\operatorname{Lip}\left(A_{t}-B_{t}\right): t=1,2, \ldots\right\} .
\end{aligned}
$$

Clearly, $\left(\mathscr{A}, d_{s}\right)$ is a complete metric space. The metric $d_{s}$ induces in $\mathscr{A}$ a topology which we call the strong topology. For each $\mathbf{A}=\left\{A_{t}\right\}_{t=1}^{\infty}$ and $\mathbf{B}=\left\{B_{t}\right\}_{t=1}^{\infty}$ in $\mathscr{A}$, we set

$$
d_{w}(\mathbf{A}, \mathbf{B})=\sup \left\{\left\|A_{t} x-B_{t} x\right\|: t=1,2, \ldots \text { and } x \in K\right\}
$$

Clearly, $\left(\mathscr{A}, d_{w}\right)$ is also a metric space. The metric $d_{w}$ induces in $\mathscr{A}$ a topology which we call the weak topology. In the sequel, for each $\mathbf{A}=\left\{A_{t}\right\}_{t=1}^{\infty} \in \mathscr{A}$, we denote

$$
\operatorname{Lip}(\mathbf{A})=\sup \left\{\operatorname{Lip}\left(A_{t}\right): t=1,2, \ldots\right\}
$$

Now we are ready to state our main result. Its proof will be given in Section 3. Section 2 is devoted to two auxiliary assertions.

THEOREM 1.1. There exists a set $\mathscr{F} \subset \mathscr{A}$ which is a countable intersection of open (in the weak topology) everywhere dense (in the strong topology) subsets of $A$ such that for each $\mathbf{C}=\left\{C_{t}\right\}_{t=1}^{\infty} \in \mathscr{F}$ and each $\epsilon>0$, the following property holds: there exist an open neighborhood $\mathcal{U}$ of $\mathrm{C}$ in $\mathscr{A}$ with the weak topology and a natural number $N$, such that for each $\mathbf{B}=\left\{B_{t}\right\}_{t=1}^{\infty} \in \mathcal{U}$, each $x, y \in K$, each integer $n \geq N$, 
and each injective mapping $r:\{1, \ldots, n\} \rightarrow\{1,2, \ldots\}$,

$$
\left\|B_{r(n)} \cdots B_{r(1)} x-B_{r(n)} \cdots B_{r(1)} y\right\|<\epsilon .
$$

A theorem of this type is called a weak ergodic theorem in the population biology literature $[6,11,13,15]$.

\section{Two auxiliary assertions}

Fix $\theta \in K$. For each $\mathbf{A}=\left\{A_{t}\right\}_{t=1}^{\infty} \in \mathscr{A}$ and each $\gamma \in(0,1)$, define $\mathbf{A}_{\gamma}=\left\{A_{t \gamma}\right\}_{t=1}^{\infty} \in$ A by

$$
A_{t \gamma} x=(1-\gamma) A_{t} x+\gamma \theta, \quad x \in K, t=1,2, \ldots
$$

It is easy to see that for each $\gamma \in(0,1)$ and each $\mathbf{A} \in \mathscr{A}$,

$$
\begin{gathered}
d_{w}\left(\mathbf{A}, \mathbf{A}_{\gamma}\right) \leq \gamma d(K), \\
d_{s}\left(\mathbf{A}, \mathbf{A}_{\gamma}\right) \leq \gamma d(K)+\gamma \sup \left\{\operatorname{Lip}\left(A_{t}\right): t=1,2, \ldots\right\} .
\end{gathered}
$$

The second inequality in (2.2) implies that the set $\left\{\mathbf{A}_{\gamma}: \mathbf{A} \in \mathscr{A}, \gamma \in(0,1)\right\}$ is everywhere dense with respect to the strong topology.

Lemma 2.1. Let $\mathbf{A}=\left\{A_{t}\right\}_{t=1}^{\infty} \in \mathcal{A}, \gamma \in(0,1)$, and $\epsilon>0$. Then, there exists a natural number $N \geq 4$ such that for each injective mapping $r:\{1, \ldots, N\} \rightarrow\{1,2, \ldots\}$ and each $x, y \in K$,

$$
\left\|A_{r(N) \gamma} \cdots A_{r(1) \gamma} x-A_{r(N) \gamma} \cdots A_{r(1) \gamma} y\right\| \leq \epsilon
$$

Proof. Let $x, y \in K$ and let $t$ be a natural number. It follows from (2.1) that

$$
\left\|A_{t \gamma} x-A_{t \gamma} y\right\| \leq(1-\gamma)\left\|A_{t} x-A_{t} y\right\| \leq(1-\gamma) \operatorname{Lip}\left(A_{t}\right)\|x-y\| .
$$

Therefore, for each natural number $t$,

$$
\operatorname{Lip}\left(A_{t \gamma}\right) \leq(1-\gamma) \operatorname{Lip}\left(A_{t}\right) .
$$

Since $\limsup \sup _{t \rightarrow \infty} \operatorname{Lip}\left(A_{t}\right) \leq 1$, it follows from (2.5) that there exists a natural number $n_{0}$ such that

$$
\operatorname{Lip}\left(A_{n \gamma}\right) \leq\left(1-\frac{\gamma}{2}\right), \quad \text { for each integer } n \geq n_{0}
$$


70 A weak ergodic theorem for infinite products

Choose an integer $n_{1} \geq 2$ such that

$$
(\operatorname{Lip}(\mathbf{A})+1)^{n_{0}}\left(1-\frac{\gamma}{2}\right)^{n_{1}} d(K) \leq \epsilon
$$

Set

$$
N=n_{0}+n_{1}+1
$$

Let the mapping $r:\{1, \ldots, N\} \rightarrow\{1,2, \ldots\}$ be injective. Define

$$
E_{1}=\left\{t \in\{1, \ldots, N\}: r(t)<n_{0}\right\}, \quad E_{2}=\{1, \ldots, N\} \backslash E_{1} .
$$

Since the mapping $r$ is injective, the cardinality

$$
\operatorname{Card}\left(E_{1}\right)<n_{0}
$$

By (2.8), (2.9), and (2.10), we have

$$
\operatorname{Card}\left(E_{2}\right)>n_{1}
$$

It follows from (1.3), (2.9), (2.6), (2.5), (1.6), (2.10), (2.11), and (2.7) that for each $x, y \in K$,

$$
\begin{aligned}
\| A_{r(N) \gamma} \cdots & A_{r(1) \gamma} x-A_{r(N) \gamma} \cdots A_{r(1) \gamma} y \| \\
& \leq \prod_{i=1}^{N} \operatorname{Lip}\left(A_{r(i) \gamma}\right)\|x-y\| \\
& \leq \prod_{i \in E_{1}} \operatorname{Lip}\left(A_{r(i) \gamma}\right) \prod_{i \in E_{2}} \operatorname{Lip}\left(A_{r(i) \gamma}\right) d(K) \\
& \leq\left(1-\frac{\gamma}{2}\right)^{\operatorname{Card}\left(E_{2}\right)} \operatorname{Lip}(\mathbf{A})^{\operatorname{Card}\left(E_{1}\right)} d(K) \\
& \leq\left(1-\frac{\gamma}{2}\right)^{n_{1}}(\operatorname{Lip}(\mathbf{A})+1)^{n_{0}} d(K) \leq \epsilon .
\end{aligned}
$$

Lemma 2.1 is proved.

Lemma 2.2. Let $\mathbf{A}=\left\{A_{t}\right\}_{t=1}^{\infty} \in \mathscr{A}, \gamma \in(0,1)$, and $\epsilon>0$. Then, there exist a natural number $N \geq 4$ and a neighborhood $\mathcal{U}$ of $\mathbf{A}_{\gamma}$ in the space $A$ with the weak topology such that for each $\mathbf{B}=\left\{B_{t}\right\}_{t=1}^{\infty} \in \mathcal{U}$, each injective mapping $r:\{1, \ldots, N\} \rightarrow$ $\{1,2, \ldots\}$, and each $x, y \in K$,

$$
\left\|B_{r(N)} \cdots B_{r(1)} x-B_{r(N)} \cdots B_{r(1)} y\right\|<\epsilon .
$$


Proof. By Lemma 2.1, there exists a natural number $N \geq 4$ such that for each injective mapping $r:\{1, \ldots, N\} \rightarrow\{1,2, \ldots\}$ and each $x, y \in K$,

$$
\left\|A_{r(N) \gamma} \cdots A_{r(1) \gamma} x-A_{r(N) \gamma} \cdots A_{r(1) \gamma} y\right\| \leq \frac{\epsilon}{8} .
$$

Choose a positive number

$$
\delta<16^{-1} \epsilon(\operatorname{Lip}(\mathbf{A})+1)^{-N}
$$

and set

$$
\mathcal{U}=\left\{\mathbf{B} \in \mathscr{A}: d_{w}\left(\mathbf{A}_{\gamma}, \mathbf{B}\right) \leq \delta\right\} .
$$

Assume that $\mathbf{B}=\left\{B_{t}\right\}_{t=1}^{\infty} \in \mathcal{U}$ and that the mapping $r:\{1, \ldots, N\} \rightarrow\{1,2, \ldots\}$ is injective. We show, by induction, that for any integer $n \in[1, N]$ and any $z \in K$,

$$
\left\|B_{r(n)} \cdots B_{r(1)} z-A_{r(n) \gamma} \cdots A_{r(1) \gamma} z\right\| \leq \delta(\operatorname{Lip}(\mathbf{A})+1)^{n} .
$$

First we show that (2.17) holds for $n=1$. Let $z \in K$. By (2.16) and the definition of $d_{w}$,

$$
\left\|B_{r(1)} z-A_{r(1) \gamma} z\right\| \leq \delta
$$

so that (2.17) is true for $n=1$. Let $z \in K, i \in\{1, \ldots, N-1\}$, and assume that (2.17) holds for $n=i$. When combined with (1.1), (1.5), (2.5), and the definition of $d_{w}$, this inductive assumption implies that

$$
\begin{aligned}
\| B_{r(i+1)} & B_{r(i)} \cdots B_{r(1)} z-A_{r(i+1) \gamma} A_{r(i) \gamma} \cdots A_{r(1) \gamma} z \| \\
\leq & \left\|B_{r(i+1)} B_{r(i)} \cdots B_{r(1)} z-A_{r(i+1) \gamma} B_{r(i)} \cdots B_{r(1)} z\right\| \\
& +\left\|A_{r(i+1) \gamma} B_{r(i)} \cdots B_{r(1)} z-A_{r(i+1) \gamma} A_{r(i) \gamma} \cdots A_{r(1) \gamma} z\right\| \\
\leq & \operatorname{Lip}\left(\mathbf{A}_{\gamma}\right)\left\|B_{r(i)} \cdots B_{r(1)} z-A_{r(i) \gamma} \cdots A_{r(1) \gamma} z\right\| \\
& +\left\|B_{r(i+1)} B_{r(i)} \cdots B_{r(1)} z-A_{r(i+1) \gamma} B_{r(i)} \cdots B_{r(1)} z\right\| \\
\leq & \operatorname{Lip}(\mathbf{A}) \delta(\operatorname{Lip}(\mathbf{A})+1)^{i}+\delta \leq \delta(\operatorname{Lip}(\mathbf{A})+1)^{i+1} .
\end{aligned}
$$

Thus, (2.17) holds for $n=i+1$ too. Therefore, (2.17) holds for $n=N$ and for any $z \in K$. Combined with (2.15), this fact implies that

$$
\left\|B_{r(N)} \cdots B_{r(1)} z-A_{r(N) \gamma} \cdots A_{r(1) \gamma} z\right\| \leq \delta(\operatorname{Lip}(\mathbf{A})+1)^{N}<\frac{\epsilon}{16} .
$$


72 A weak ergodic theorem for infinite products

Now let $x, y \in K$. It follows from (2.20) and the definition of $N$ (see (2.14)) that

$$
\begin{aligned}
\| B_{r(N)} & \cdots B_{r(1)} x-B_{r(N)} \cdots B_{r(1)} y \| \\
\leq & \left\|A_{r(N) \gamma} \cdots A_{r(1) \gamma} x-A_{r(N) \gamma} \cdots A_{r(1) \gamma} y\right\| \\
& +\left\|B_{r(N)} \cdots B_{r(1)} x-A_{r(N) \gamma} \cdots A_{r(1) \gamma} x\right\| \\
& +\left\|B_{r(N)} \cdots B_{r(1)} y-A_{r(N) \gamma} \cdots A_{r(1) \gamma} y\right\| \\
\leq & \frac{2 \epsilon}{16}+\frac{\epsilon}{8}<\epsilon .
\end{aligned}
$$

Lemma 2.2 is proved.

\section{Proof of Theorem 1.1}

Let $\mathbf{A}=\left\{A_{t}\right\}_{t=1}^{\infty} \in \mathscr{A}, \gamma \in(0,1)$, and let $n$ be a natural number. By Lemma 2.2, there exist an open neighborhood $\mathcal{U}(\mathbf{A}, \gamma, n)$ of $\mathbf{A}_{\gamma}$ in $\mathscr{A}$ with the weak topology and a natural number $N(\mathbf{A}, \gamma, n)$ such that the following property holds:

(i) for each $\mathbf{B}=\left\{B_{t}\right\}_{t=1}^{\infty} \in \mathcal{U}(\mathbf{A}, \gamma, n)$, each injective mapping

$$
r:\{1, \ldots, N(\mathbf{A}, \gamma, i)\} \longrightarrow\{1,2, \ldots\}
$$

and each $x, y \in K$, we have

$$
\left\|B_{r(N(\mathbf{A}, \gamma, n))} \cdots B_{r(1)} x-B_{r(N(\mathbf{A}, \gamma, n))} \cdots B_{r(1)} y\right\| \leq \frac{1}{n} .
$$

Define

$$
\mathscr{F}=\bigcap_{n=1}^{\infty} \cup\{\mathcal{A}(\mathbf{A}, \gamma, i): \mathbf{A} \in \mathscr{A}, \gamma \in(0,1), i \geq n\} .
$$

Clearly, $\mathscr{F}$ is a countable intersection of open (in the weak topology) everywhere dense (in the strong topology) subsets of $\mathscr{A}$.

Let $\mathbf{C}=\left\{C_{t}\right\}_{t=1}^{\infty} \in \mathscr{F}$ and $\epsilon>0$. We may assume that $\epsilon<1$. Choose a natural number

$$
q>\frac{8}{\epsilon} .
$$

By the definition of $\mathscr{F}$, there exist $\mathbf{A} \in \mathscr{A}, \gamma \in(0,1)$, and an integer $i \geq q$ such that

$$
\mathbf{C} \in \mathcal{U}(\mathbf{A}, \gamma, i) .
$$

Let $\mathbf{B}=\left\{B_{t}\right\}_{t=1}^{\infty} \in \mathcal{U}(\mathbf{A}, \gamma, i)$. It follows from the definition of $\mathcal{U}(\mathbf{A}, \gamma, i)$ and property (i) that for each injective mapping $r:\{1, \ldots, N(\mathbf{A}, \gamma, i)\} \rightarrow\{1,2, \ldots\}$ and each $x, y \in K$, we have

$$
\left\|B_{r(N(\mathbf{A}, \gamma, i))} \cdots B_{r(1)} x-B_{r(N(\mathbf{A}, \gamma, i))} \cdots B_{r(1)} y\right\| \leq \frac{1}{i} \leq \frac{1}{q}<\epsilon .
$$


This implies that for each $x, y \in K$, each integer $n \geq N(\mathbf{A}, \gamma, i)$, and each injective mapping $r:\{1, \ldots, n\} \rightarrow\{1,2, \ldots\}$, we have

$$
\left\|B_{r(n)} \cdots B_{r(1)} x-B_{r(n)} \cdots B_{r(1)} y\right\|<\epsilon .
$$

This completes the proof of Theorem 1.1.

\section{Acknowledgments}

The work of the first author was partially supported by the Israel Science Foundation founded by the Israel Academy of Sciences and Humanities (grant 592/00), by the fund for the Promotion of Research at the Technion, and by the Technion Vice President for Research (VPR) fund.

\section{References}

[1] I. Amemiya and T. Andô, Convergence of random products of contractions in Hilbert space, Acta Sci. Math. (Szeged) 26 (1965), 239-244.

[2] H. H. Bauschke, A norm convergence result on random products of relaxed projections in Hilbert space, Trans. Amer. Math. Soc. 347 (1995), no. 4, 1365-1373.

[3] H. H. Bauschke and J. M. Borwein, On projection algorithms for solving convex feasibility problems, SIAM Rev. 38 (1996), no. 3, 367-426.

[4] H. H. Bauschke, J. M. Borwein, and A. S. Lewis, The method of cyclic projections for closed convex sets in Hilbert space, Recent Developments in Optimization Theory and Nonlinear Analysis (Jerusalem, 1995), Contemp. Math., vol. 204, American Mathematical Society, Rhode Island, 1997, pp. 1-38.

[5] H. Brézis and P.-L. Lions, Produits infinis de résolvantes, Israel J. Math. 29 (1978), no. 4, 329-345 (French).

[6] J. E. Cohen, Ergodic theorems in demography, Bull. Amer. Math. Soc. (N.S.) 1 (1979), no. 2, 275-295.

[7] F. S. De Blasi and J. Myjak, Sur la convergence des approximations successives pour les contractions non linéaires dans un espace de Banach, C. R. Acad. Sci. Paris Sér. A-B 283 (1976), no. 4, 185-187 (French).

[8] J. Dye, M. A. Khamsi, and S. Reich, Random products of contractions in Banach spaces, Trans. Amer. Math. Soc. 325 (1991), no. 1, 87-99.

[9] J. Dye, T. Kuczumow, P.-K. Lin, and S. Reich, Convergence of unrestricted products of nonexpansive mappings in spaces with the Opial property, Nonlinear Anal. 26 (1996), no. 4, 767-773.

[10] J. Dye and S. Reich, Random products of nonexpansive mappings, Optimization and Nonlinear Analysis (Haifa, 1990), Pitman Research Notes in Mathematics Series, vol. 244, Longman Scientific \& Technical, Harlow, 1992, pp. 106-118.

[11] T. Fujimoto and U. Krause, Asymptotic properties for inhomogeneous iterations of nonlinear operators, SIAM J. Math. Anal. 19 (1988), no. 4, 841-853.

[12] P.-K. Lin, Unrestricted products of contractions in Banach spaces, Nonlinear Anal. 24 (1995), no. 7, 1103-1108.

[13] R. D. Nussbaum, Some nonlinear weak ergodic theorems, SIAM J. Math. Anal. 21 (1990), no. 2, 436-460.

[14] S. Reich, The alternating algorithm of von Neumann in the Hilbert ball, Dynam. Systems Appl. 2 (1993), no. 1, 21-25. 
74 A weak ergodic theorem for infinite products

[15] S. Reich and A. J. Zaslavski, Convergence of generic infinite products of nonexpansive and uniformly continuous operators, Nonlinear Anal. 36 (1999), no. 8, Ser. A: Theory Methods, 1049-1065.

[16] Convergence of generic infinite products of order-preserving mappings, Positivity 3 (1999), no. 1, 1-21.

[17] Convergence of Krasnoselskii-Mann iterations of nonexpansive operators, Math. Comput. Modelling 32 (2000), no. 11-13, 1423-1431.

[18] Infinite products of resolvents of accretive operators, Topol. Methods Nonlinear Anal. 15 (2000), no. 1, 153-168.

Simeon Reich: Department of Mathematics, The Technion-Israel Institute of Technology, 32000 Haifa, Israel

E-mail address: sreich@tx.technion.ac.il

Alexander J. Zaslavski: Department of Mathematics, The Technion-Israel Institute of Technology, 32000 Haifa, Israel

E-mail address: ajzasl@tx.technion.ac.il 


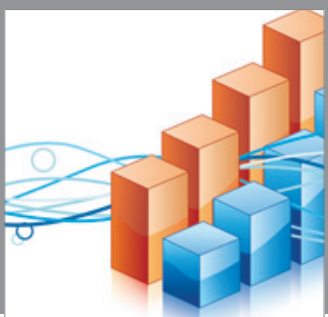

Advances in

Operations Research

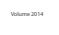

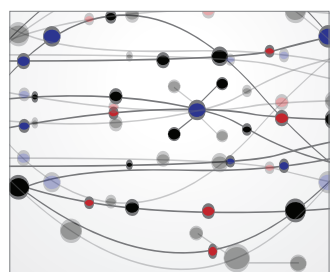

\section{The Scientific} World Journal
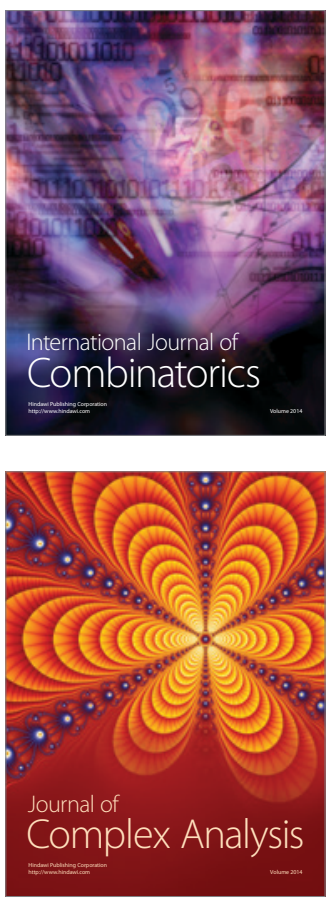

International Journal of

Mathematics and

Mathematical

Sciences
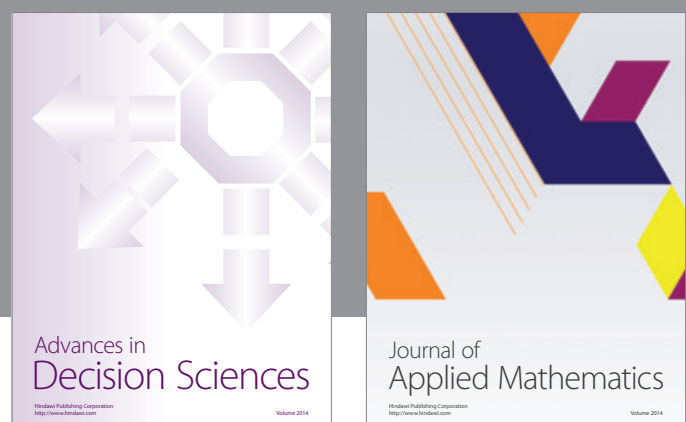

Journal of

Applied Mathematics
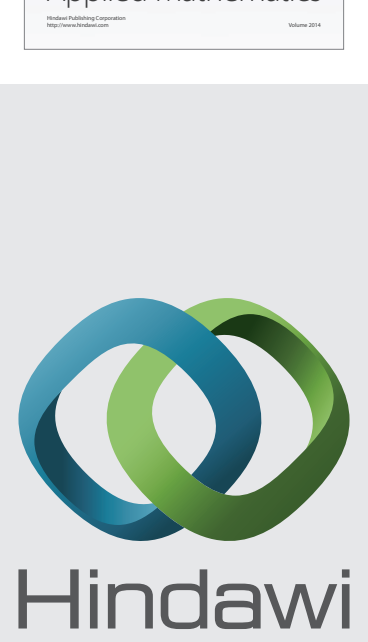

Submit your manuscripts at http://www.hindawi.com
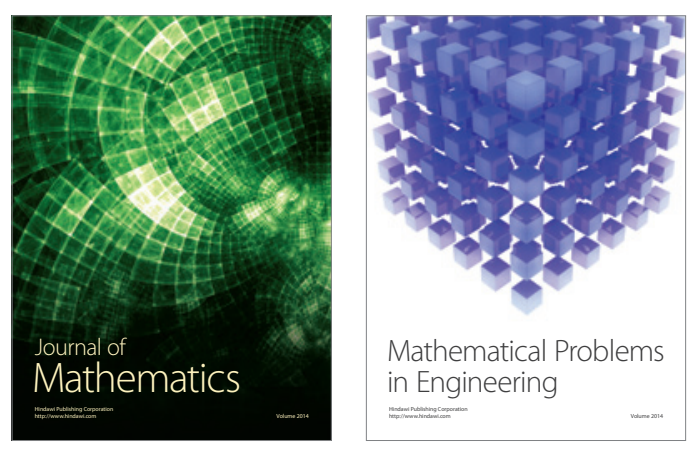

Mathematical Problems in Engineering
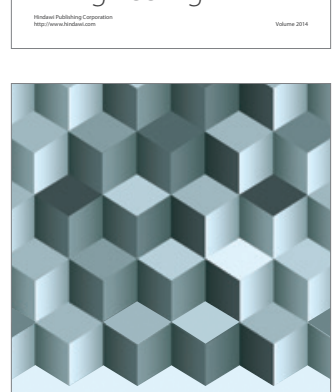

Journal of

Function Spaces
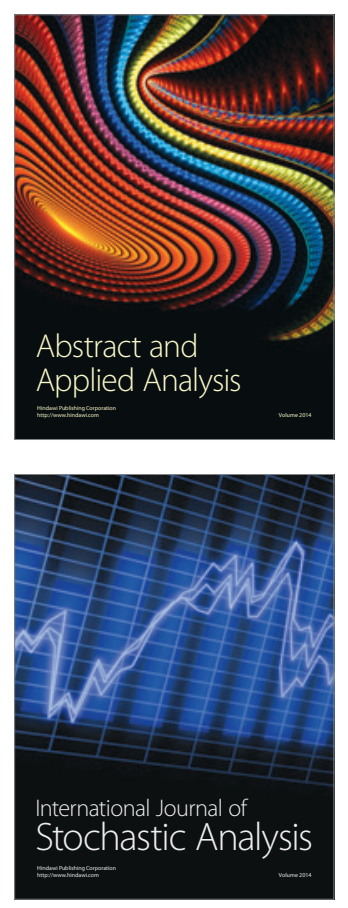

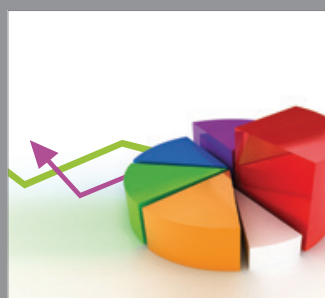

ournal of

Probability and Statistics

Promensencen
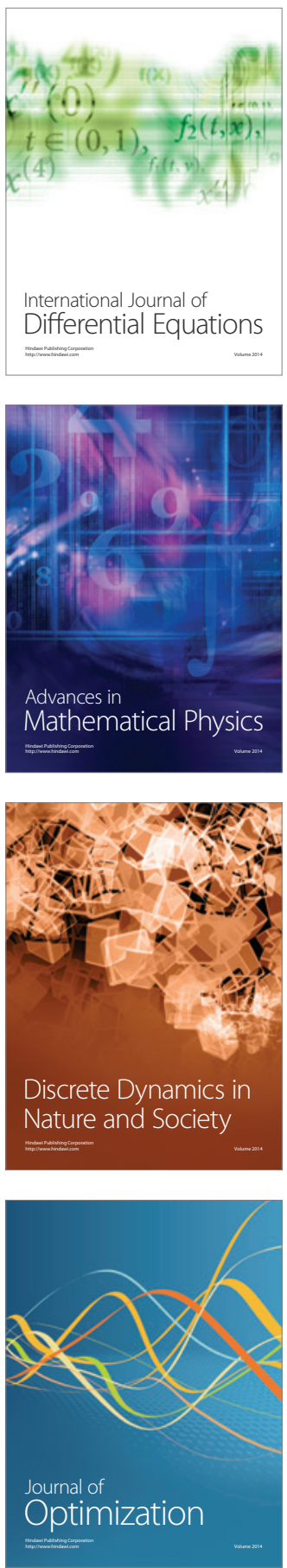